\title{
TREATMENT OF COLIFORM MASTITIS IN BOVINE PRACTICE: - CAN ANTIBIOTICS BE AVOIDED?
}

\author{
Jørgen Katholm \\ Søndergade 37, Vivild, DK-8961, Allingaabro Denmark.
}

\begin{abstract}
Background
Coliform mastitis is an acute and potentially lethal type of mastitis in bovine practice. The therapeutical approach to acute coliform mastitis is frequently discussed. While is generally accepted that supportive therapy, such as fluid therapy, is necessary, the administration of antibiotics is questionned. This is due to mainly two reasons. Firstly, the clinical ill effects observed in coliform mastitis are often thought to be due to the effect of the accompanying endotoxicosis, which would not be influenced positively by the killing of the non-invasive E. coli. Secondly, antibiotics effective against E. coli, are potent, broadspectered antibiotics, with heavy impact on the micro-ecology. The unnecessary use of such antibiotics would subsequently contribute to the development of antibiotic resistance.
\end{abstract}

\begin{abstract}
Aim
The aim of the present investigation was to determine the clinical outcome of two standard treatment regimens, one including antibiotics and one without.
\end{abstract}

\section{Material and methods}

During a 3 year period, cows presenting the following clinical signs: swelling of the quarter, acute onset, reduced feed intake and milk yield and no distinct changes of the milk, were treated for clinical coliform mastitis. The standard treatment regimen for all cows included the intravenous administration of oxytocin, calcium, hypertonic fluid, invertose and NSAID. Pain relief was obtained by the application of an epidural analgesia containing xylazin and morphine. The number of $\mathrm{CFU} / \mathrm{cm}^{2}$ of the milk was determined by direct culture on blood agar plates in the practice laboratory. All cows were examined, treated and sampled again 24 hours later. On day 21, clinical status, milk yield and the number of culled or dry teats were recorded for all cows. The first year, all cows were treated without the use of antibiotics (group 1), later cases were treated either with danofloxacin iv or cefquinome imm (group2).

\section{Statistics}

The number of clinically restored cows, number of cows with reduced milk yield and number of cows with culled or dry teats in groups 1 and 2 were counted. All numbers in the respective groups were compared by Fisher's exact test.

\section{Results and conclusion}

Fifty-six cows entered the study, and 28 of these were treated without antibiotics. Of the remaining 28 cows, 19 were treated with danofloxacin and 9 with cefquinome. It appeared that cows with 142 $\mathrm{CFU} / \mathrm{cm} 2$ or less, recovered without antibiotics. The use of antibiotics in cows with $142<\mathrm{CFU} / \mathrm{cm}^{2}$ 
$<$ app. $200 \mathrm{CFU} / \mathrm{cm}^{2}$ in the milk, was beneficial. Cows with milk containing more than app. $200 \mathrm{CFU}$ $/ \mathrm{cm}^{2}$ were generally severely ill and difficult to save, antibiotics or not. The routine determination of $\mathrm{CFU} / \mathrm{cm}^{2}$ in coliform mastitis is therefore an important decision support tool, if the use of antibiotics in the treatment of cows with coliform mastitis is to be avoided.

\title{
AMOXICILLIN PLASMA CONCENTRATIONS AFTER PER ORAL APPLICATION IN NEWLY WEANED PIGS - EFFECTS OF ESCHERICHIA COLI O149,F4 ASSOCIATED DIARRHOEA
}

\author{
Gerda Majgaard Jensen ${ }^{1}$, Jens Lykkesfeldt ${ }^{2}$ and Ove Svendsen ${ }^{2}$ \\ ${ }^{1}$ Department of Clinical Studies, ${ }^{2}$ Depamrtment of Pharmacology and Pathobiology, The Royal \\ Veterinary and Agricultural University, Bülowsvej 13, DK-1870 Frederiksberg C, Denmark.
}

The presence of a pathological condition often causes a change in tissue permeability and blood supply. Penetration of an antibiotic into an organ is dependent on those two factors. It is therefore interesting to test if a pathological condition is altering the absorption of an antibiotic into the diseased organ. In this study it was tested if the absorption of Amoxicillin from the alimentary tract could be altered by an Escherichia coli O149,F4 associated diarrhoea.

Twenty-four healthy 4 weeks old newly weaned pigs where used. 14 were inoculated with $10^{10} \mathrm{cfu}$ of a pathogen O149,F4 strain and responded with a watery diarrhoea 12-16 hrs. later. Having diarrhoea the pigs were treated through a gastric tube with an Amoxicillin trihydrate solution to achieve a dosis of $20 \mathrm{mg}$ pr. kg. bdwgt. A control group of 10 pigs without any signs of diarrhoea was treated as well. Blood samples where taken at 0,30,60, 90, $120 \mathrm{~min}$. and 3,6,12 and $24 \mathrm{hrs}$ after medication. The concentration of Amoxicillin in plasma where analysed by a high performance liquid chromatography.

Preliminary data shows a difference in Amoxicillin plasma concentration between the diarrhoeic and control group. 